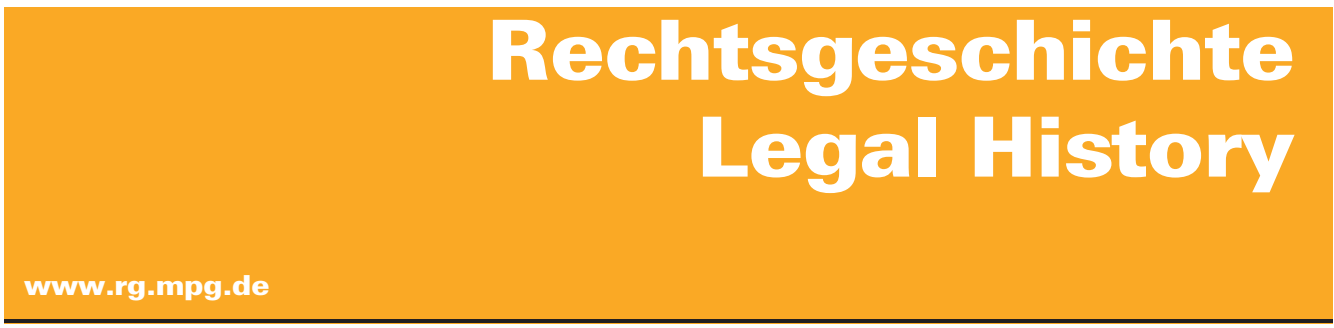

http://www.rg-rechtsgeschichte.de/rg23

$R g 232015 \quad 268-269$

Zitiervorschlag: Rechtsgeschichte - Legal History Rg 23 (2015)

http://dx.doi.org/10.12946/rg23/268-269

\title{
Tilman Repgen
}

$»$ Nur schildern, wie es war« 


\section{Tilman Repgen}

\section{»Nur schildern, wie es war«}

1. Noch mehr als um ein Gegen- oder Miteinander von Normen-, Wissenschafts- und Praxisgeschichte geht es Oestmann um eine ihm wichtige methodische Regel, die man knapp so zusammenfassen könnte: Nachsubsumtionen sind nicht erlaubt! Der heutige Strafrechtsprofessor darf nicht ein Fehlurteil im frühen 19. Jahrhundert diagnostizieren. Oestmann schreibt: »Der Rechtshistoriker darf nicht einfach sein juristisches Handwerkszeug auspacken und eigenständig damit beginnen, Normen der Vergangenheit selbst auszulegen« (nach Fn. 9).

Thukydides schrieb in seiner Geschichte des peloponnesischen Krieges unter anderem über das Jahr 430 v. Chr., in dem in Athen die Pest ausbrach. Die Seuche sei von Ägypten gekommen, so berichtet er, die Ärzte hätten ihr machtlos gegenübergestanden. Dann heißt es dort:

»Mag nun jeder darüber sagen, Arzt oder Laie, was seiner Meinung nach wahrscheinlich der Ursprung davon war und welchen Ursachen er eine Wirkung bis in solche Tiefe zutraut. Ich will nur schildern, wie es war; nur die Merkmale, an denen man sie am ehesten wiedererkennen könnte, um dann Bescheid zu wissen, wenn sie je noch einmal hereinbrechen sollte. Die will ich darstellen, der ich selbst krank war und selbst andere leiden sah. ${ }^{1}$

In klassischer Weise ist damit die Aufgabe von Geschichtsschreibung umrissen. Indirekt knüpft Oestmann seine Botschaft daran an, wenn er sich gleich zu Beginn auf die berühmte Einleitung Rankes zu seinen "Geschichten der romanischen und germanischen Völker« von 1824 beruft, wo man liest, er, Ranke, wolle nicht über die Vergangenheit richten, sondern »bloß sagen, wie es eigentlich gewesen«(S. VI). Ranke bestreitet an dieser Stelle zwar nicht die Möglichkeit eines
»Richtens « über die Vergangenheit, ${ }^{2}$ aber er macht das Richten nicht zu seinem Programm, sondern sucht mit seinem Buch einen »Weg zu einer vollständigeren Einsicht in die Geschichte« (S. V). Ranke spricht hier wohlgemerkt über seine "Absicht«, die Zielsetzung seines Buches, nicht - oftmals verkannt - über seine Methode.

Wenn man sich, wofür vieles spricht, darauf einigen möchte, dass das "Richten « nicht die Aufgabe des Rechtshistorikers ist, so bleibt das Streben nach dieser Einsicht. Das hat mit Verstehen zu tun. Was man nicht begreifen kann, darin hat man auch keine Einsicht. Das Nachvollziehen von Handlungen, auch von rechtlichen Entscheidungen, gehört daher m. E. durchaus zur Aufgabe des Rechtshistorikers. Das bringt es mit sich, dass die logische Konsistenz der Quellen, ihre innere, gegebenenfalls auch: fachwissenschaftliche Stimmigkeit mit in die Interpretation einzubeziehen ist. Wichtig ist und hierin liegt das Problem, dass die Maßstäbe solcher Quelleninterpretation nicht anachronistisch gewählt werden dürfen. Es trifft zwar zu, dass beispielsweise die Geschlechtsvormundschaft des älteren römischen Rechts nicht mit dem menschenrechtlichen Gleichheitssatz vereinbar ist, aber das ist wegen des Anachronismus keine historische Einsicht, sondern eine rechtstheoretische. Es ist keine vernünftige historische Methode, antikes römisches Recht an modernen Menschenrechtskonventionen zu messen. Man könnte es aber sehr wohl mit antiken Vorstellungen von der gleichen Menschenwürde konfrontieren, wie es bezüglich der Sklaverei geschieht.

Möchte man z.B. eine Gerichtsentscheidung aus dem 18. Jh. verstehen (Einsicht im Sinne Rankes gewinnen), so bleibt nichts anderes übrig, als auch zu fragen, ob das Verfahren nach damaligen rechtlichen Maßstäben korrekt verlaufen ist. Man mag diese Frage nicht immer beantworten können, weil die Rechtslage nicht aufklärbar ist usw.,
1 Thukydides, Geschichte des Peloponnesischen Krieges, 1. Teil: Buch I-IV, griechisch-deutsch, übersetzt und mit einer Einführung und Erläuterung versehen von Georg Peter Landmann, Zürich 1993, Buch II, 48, S. $251 \mathrm{ff}$. (Hervorhebungen von TR).
2 Ob Ranke dasselbe damit meint wie Oestmann, wäre noch erst zu untersuchen. 
aber es ist nicht zu sehen, dass allein die Tatsache der zeitlichen Distanz zu dem Urteil die Möglichkeit dazu verstellt. Mit der zeitlichen Entfernung wächst allerdings in der Regel die Schwierigkeit, die zeitgenössischen Maßstäbe zu erfassen. Manches entzieht sich auch gänzlich einem historischen Nachvollziehen. So ist die "Richtigkeit" einer Beweiswürdigung weithin unüberprüfbar. Das ist aber nun wiederum kein Problem rein zeitlicher Art, sondern gilt auch für die aktuelle Rechtspraxis, bei der sich die Juristen nicht scheuen, eine normative Überprüfung vorzunehmen, dies sogar als ihre Aufgabe betrachten. Solche Nachprüfung zielt nicht auf »nachträgliche Harmonisierungen « und ändert gewiss nichts an den Tatsachen, aber sie vervollständigt die Einsicht in das Geschehen. Die Wimmelbilder der Vergangenheit bleiben. Das große, klare und einheitliche Gemälde ist nicht zu erhoffen. Bei einer normativen Überprüfung kann es nicht darum gehen, den geschichtlichen Gestalten gleichsam Schulnoten zu geben. Der Erkenntniswert eines solchen Unterfangens wäre mindestens fragwürdig. Aber warum sollte es nicht möglich sein, vergangenes Unrecht als solches zu erkennen und damit vollständigere Einsicht in die Geschichte zu gewinnen? Für die juristische Zeitgeschichte ist das bislang nicht in Zweifel gezogen worden. Noch einmal: die zeitliche Distanz mag die Aufgabe erschweren, aber sie macht sie - so überhaupt Quellen vorliegen - nicht unmöglich. Ob die normative Überprüfung überzeugt, ist selbstverständlich, wie jede historische Arbeit, der Kritik ausgesetzt, aber letztlich eine Frage methodisch ordentlicher Durchführung.

2. In einem inneren Zusammenhang mit der Frage nach einer nachträglichen Überprüfung von Gerichtsverfahren steht der zweite Kernpunkt Oestmanns: das Verhältnis von Normengeschichte zur Praxisgeschichte. Eine logische Hierarchie in dem Sinne, dass zuerst die Normen und dann die Praxis beleuchtet werden müssten, ist in dieser Allgemeinheit ebenso wenig begründbar wie die Annahme, Praxisgeschichte könne ohne Normengeschichte (oder umgekehrt) gar nicht geschrieben werden. $\mathrm{Ob}$ das eine zum anderen kommen muss, ist vielmehr eine Frage des Erkenntnisziels. Selbstverständlich kann man in interessanter Weise Rechtsgeschichte betreiben, ohne dabei positives Recht zu betrachten. Wenn man - wie der eingangs erwähnte Strafrechtsprofessor - wissen möchte, ob Urteil und zugrundeliegendes
Prozessrecht zueinander passen, ist nicht $\mathrm{zu}$ sehen, warum man diese Frage nicht stellen dürfte. Der normative Maßstab muss nur historisch zutreffend bestimmt werden. Dass man aber überhaupt zu normativen Aussagen über lange Vergangenes kommen kann, räumt auch Oestmann ein, da die Erkenntnis von »Brüchen und Widersprüchen« (nach Fn. 23) auch dem Historiker zugänglich sein soll. Dazu braucht man einen Maßstab, eine Norm. Das Recht selbst ist weder auf die eine noch die andere Weise vollständig zu ergründen, sondern - das ist methodisch wichtig - die Reichweite einer Geschichte über das Recht hängt immer auch von den zu Rate gezogenen Quellen ab.

Forschungsfragen konditionieren den Gegenstand der wissenschaftlichen Tätigkeit. Darin ist man frei. Niemand zweifelt daran, auch wenn es extrinsische Faktoren gibt, die die Forschungsinteressen beeinflussen. Das ist bei jedem Freiheitsgebrauch der Fall und kein Grund, die Freiheit selbst in Zweifel zu ziehen. Es besteht insoweit überhaupt keine Rechtfertigungsnotwendigkeit.

Der Begriff »Methode« steht hingegen für die wissenschaftlich anerkannten Verfahren, mit denen akzeptable Antworten auf die Forschungsfragen gefunden werden können. Solche Regeln müssen den Denkgesetzen gehorchen und sind nicht frei definierbar. Die Auswahl der Fragestellung ist zunächst einmal von der Methode ihrer Beantwortung zu trennen. So kann man etwa versuchen, Verfassungsgeschichte mit soziologischen Methoden zu betreiben, aber das ist nicht zwingend. Oder man kann Praxisgeschichte mit quantitativen Methoden betreiben, aber durchaus auch ohne diese. Wer aber die Frage schon so stellt, dass ihn z. B. die theologischen Hintergründe der Entstehung der modernen Vertragslehre interessieren, kommt nicht umhin, die Theologiegeschichte einzubeziehen.

Methoden und Forschungsfragen sind also nicht voneinander isoliert, aber sie lassen sich unterscheiden. Ein Gegeneinander von Normen-, Wissenschafts- und Praxisgeschichte wäre ohne Sinn. Die diesen Geschichten zugeordneten Quellengruppen sind vielfältig verschränkt, können aber auch isoliert Gegenstand der Forschung mit je eigenem Erkenntniswert werden. 